\title{
Caractéristiques des utilisateurs de cigarettes électroniques et leurs perceptions des avantages, des dangers et des risques de la cigarette électronique : résultats d'un sondage auprès d'un échantillon de commodité à Ottawa (Canada)
}

K. D. Volesky, M.A., M. Sc. (1); A. Maki, M. Sc. (1); C. Scherf, M. Sc. (1); L. M. Watson, M. Sc. (1); E. Cassol, Ph. D. (1); P. J. Villeneuve, Ph. D. (1, 2)

Cet article a fait l'objet d'une évaluation par les pairs.

Diffuser cet article sur Twitter

\section{Résumé}

Introduction : Bien que l'utilisation de la cigarette électronique (« vapotage ») soit en hausse au Canada, peu d'efforts ont été consacrés à la description des utilisateurs de cigarettes électroniques («vapoteurs »). C'est dans ce contexte que nous avons mené une étude à Ottawa (Canada) afin de décrire les perceptions qu'ont les utilisateurs de cigarettes électroniques des avantages, des dangers et des risques de ces dernières. Nous avons également recueilli de l'information pour savoir pourquoi, comment et où ils utilisent la cigarette électronique ainsi que sur les effets secondaires.

Méthodologie : Un sondage en ligne de 24 questions a été soumis à des personnes ayant acheté des cigarettes électroniques ou des fournitures connexes dans l'un des 17 commerces de cigarettes électroniques à Ottawa. On a caractérisé les répondants au moyen d'analyses descriptives, puis nous avons appliqué des modèles de régression logistique pour évaluer la relation entre ces caractéristiques et la perception par les répondants des dangers de la cigarette électronique.

Résultats : Lâge moyen des 242 répondants était de 38,1 ans (plage : 16 à 70 ans) et, de ce nombre, 66 \% étaient des hommes. Près de la totalité $(97,9 \%)$ des répondants avaient fumé 100 cigarettes ou plus au cours de leur vie. Plus de $80 \%$ des répondants ont indiqué que la volonté d'arrêter de fumer constituait l'une des principales raisons de recourir à la cigarette électronique, et $60 \%$ ont mentionné qu'ils avaient l'intention de cesser l'utilisation de la cigarette électronique un jour. Environ $40 \%$ des répondants ont fait état d'effets secondaires au cours des 2 heures suivant l'utilisation des cigarettes électroniques. Les répondants ayant signalé n'avoir ressenti aucun des effets secondaires énumérés étaient environ 3,2 fois plus nombreux à ne percevoir aucun danger dans la cigarette électronique que les personnes ayant signalé des effets secondaires (rapport de cotes $=3,17$; intervalle de confiance à $95 \%: 1,75$ à 5,73 ).

Conclusion : D’après nos constatations, la majorité des utilisateurs de cigarettes électroniques ont recours à ces dernières pour réduire ou cesser leur consommation de tabac et ils les perçoivent comme inoffensives. Étant donné que nous avons utilisé un échantillonnage de commodité, le lecteur doit faire preuve de prudence dans la généralisation de nos constatations à tous les utilisateurs de cigarettes électroniques au Canada.
Points saillants

- Cette étude décrit les perceptions des utilisateurs de cigarettes électroniques et fournit de l'information sur ces utilisateurs et sur les effets secondaires qu'ils ont pu ressentir.

- Près de la totalité des 242 répondants (environ $98 \%$ ) avaient fumé 100 cigarettes ou plus au cours de leur vie.

- Plus de $80 \%$ des répondants ont dit que l'abandon définitif du tabac ou la réduction du nombre de cigarettes fumées faisaient partie des principales raisons les ayant poussés à commencer à utiliser la cigarette électronique.

- Environ $40 \%$ des répondants ont dit avoir ressenti au moins 1 effet secondaire au cours des 2 heures ayant suivi l'utilisation d'une cigarette électronique.

- Soixante pour cent $(60 \%)$ des répondants sont d'avis que la cigarette électronique est inoffensive.

Mots-clés : cigarettes électroniques, abandon du tabac, nicotine, tabagisme, produits du tabac, perception

Rattachement des auteurs :

1. Faculté des sciences de la santé, Université Carleton, Ottawa (Ontario), Canada 2. École de santé publique Dalla Lana, Université de Toronto, Toronto (Ontario), Canada

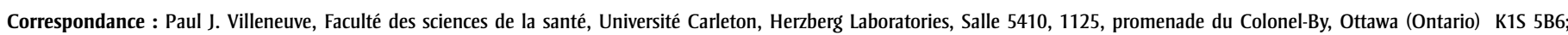
tél. : 613-520-2600, poste 3359; courriel : paul.villeneuve@carleton.ca 


\section{Introduction}

Les cigarettes électroniques sont des dispositifs alimentés par piles qui réchauffent une solution liquide pour en faire une brume en aérosol que les utilisateurs (« vapoteurs ») inhalent, reproduisant ainsi le geste de porter une cigarette à la bouche $^{1}$. La solution liquide (appelée " liquide à vapoter ») est généralement composée de propylèneglycol, de glycérine, d'aromatisant et de nicotine $e^{2,3,4}$. Contrairement à la cigarette, la cigarette électronique ne produit pas de fumée secondaire indirecte ${ }^{5}$, et des études sur l'exposition suggèrent qu'elle ne contient pas les mêmes taux de produits chimiques nocifs que la cigarette ${ }^{2,4,5,6}$. Cependant, les vapeurs produites par la cigarette électronique pourraient augmenter les concentrations de poussières fines en suspension dans les lieux clos au point d'avoir des effets sur la santé ${ }^{7}$. Les effets à court et à long terme sur la santé, tant pour les utilisateurs de cigarettes électroniques que pour leur entourage, ne sont pas bien compris, et combler cette lacune en matière de recherche constitue une priorité $^{1,2,4,5,8}$.

La popularité et la vente des cigarettes électroniques ont considérablement augmenté depuis l'arrivée de ces dernières sur le marché nord-américain en $2007^{9,10}$. On craint que les personnes qui n'ont jamais fumé et les jeunes soient incités à fumer à cause de la cigarette électronique, et que d'ex-fumeurs reprennent le tabagisme ${ }^{1,11}$. D’après les études publiées à ce jour, la majorité des vapoteurs sont des fumeurs ou d'ex-fumeurs de cigarettes $^{12-16}$, ils ont recours à la cigarette électronique pour réduire ou cesser leur consommation de cigarettes et ils perçoivent la cigarette électronique comme étant moins nocive que les cigarettes ${ }^{13,16,17}$. Bien qu'il ait été prouvé que les cigarettes électroniques permettent de réduire ou de cesser la consommation de tabac, cette constatation ne fait pas l'unanimité ${ }^{18,19}$.

Malgré l'augmentation de la sensibilisation à la cigarette électronique et de son utilisation au Canada, peu d'études ont été consacrées à la description des vapoteurs ${ }^{9,12,20,21}$. Un sondage en direct portant sur 1188 jeunes adultes a révélé que $43 \%$ d'entre eux connaissaient l'existence de la cigarette électronique et que 5,7 \% l'utilisaient ${ }^{12}$. Cette même étude a aussi révélé que $80 \%$ des fumeurs de cigarette avouaient être tentés d'essayer la cigarette électronique, soit pour les aider à cesser de fumer, soit pour en faire usage dans les lieux où il est interdit de fumer ${ }^{12}$. L'Enquête canadienne sur le tabac, l'alcool et les drogues de 2013 a révélé que 8,5 \% des Canadiens de 15 ans et plus avaient déjà essayé la cigarette électronique $^{21}$, mais peu de données canadiennes permettent de savoir pourquoi, où et comment la cigarette électronique est utilisée et de connaître les perceptions qu'en ont ses utilisateurs.

L'augmentation à la fois de l'utilisation de la cigarette électronique et de sa notoriété ainsi que le manque de données canadiennes sur les vapoteurs font ressortir la nécessité de mieux décrire les utilisateurs de cigarette électronique. (Au moment d'entreprendre la présente étude, on ne disposait d'aucune donnée sur les vapoteurs à Ottawa.) Il est important de disposer de données locales, en raison du rôle que jouent les autorités locales dans la mise en œuvre de politiques sur le tabagisme et sur l'utilisation de la cigarette électronique.

Cette étude vise à examiner pourquoi, comment et où la cigarette électronique est utilisée ainsi que les perceptions des avantages, des dangers et des risques des cigarettes électroniques chez un échantillon de vapoteurs de la région d'Ottawa. Dans la mesure où les perceptions qu'ont les vapoteurs des effets nocifs de la cigarette électronique pourraient avoir une incidence sur la fréquence d'utilisation de cette dernière et sur la décision d'en faire usage en présence d'autres personnes, cette étude évalue aussi si les caractéristiques des vapoteurs varient en fonction des perceptions voulant que la cigarette électronique soit inoffensive.

\section{Méthodologie}

Nous avons utilisé un sondage en ligne de 24 questions (accessible sur demande auprès des auteurs) pour recueillir des renseignements sur les utilisateurs de cigarettes électroniques : qui sont les vapoteurs, comment, pourquoi et où ils vapotent et quelles sont leurs perceptions des avantages, des dangers et des risques de l'utilisation de la cigarette électronique. Les personnes ayant effectué un achat dans l'un des 17 commerces de cigarettes électroniques à Ottawa étaient admissibles au sondage. Le recrutement de répondants par l'entremise des commerces de cigarettes électroniques de la région d'Ottawa s'est révélé la meilleure façon d'approcher efficacement la population cible, à savoir les personnes résidant dans la région d'Ottawa qui font usage de cigarettes électroniques.

Les répondants ont été recrutés entre le 8 janvier et le 2 mars 2015, et ce, de deux façons. En premier lieu, 2364 imprimés en format de carte d'affaires avec un lien vers le sondage en ligne ont été distribués aux acheteurs dans les commerces de cigarettes électroniques de la région d'Ottawa. En second lieu, ces commerces ont affiché le lien vers le sondage sur la page Facebook de leur entreprise. Les répondants n'ont pas eu à fournir leurs coordonnées et leurs adresses IP n'ont pas été recueillies. Les répondants ayant choisi de fournir leur adresse électronique ont été inscrits à un tirage de quatre cartes-cadeaux Tim Hortons d'une valeur de 25 \$ chacune.

L'étude a été approuvée sur le plan éthique par le Comité d'éthique de la recherche de l’Université Carleton.

\section{Mesures}

Le sondage en quatre parties a été administré à l'aide du logiciel de sondage Qualtrics (http://www.qualtrics.com/ research-suite $)^{22}$. Les questions de la section des données sociodémographiques portaient sur l'âge, le sexe, la situation de famille, l'appartenance à une minorité visible, l'emploi, les études et le revenu familial des répondants.

Dans la section sur le mode de vie, on demandait aux participants s'ils avaient fumé 100 cigarettes ou plus au cours de leur vie, le nombre d'années de tabagisme et la quantité de cigarettes fumées (pendant leur période de tabagisme), leur perception de leur état de santé général et la fréquence de leur consommation d'alcool ainsi que de 
leur activité physique. Le sondage n'a pas évalué leur situation quant à la consommation de cigarettes. Une bonne partie des questions sociodémographiques et sur le mode de vie ont été adaptées de l'Enquête sur la santé dans les collectivités canadiennes de $2012^{23}$.

Dans la section sur l'utilisation de la cigarette électronique, on a demandé aux répondants dans quel contexte ils avaient entendu parler de la cigarette électronique pour la première fois, quand ils avaient commencé à l'utiliser, combien de fois ils l'utilisaient au cours d'une seule " séance ", leur consommation de nicotine et de liquide à vapoter, les effets secondaires ressentis, le type de cigarette électronique utilisé (question ouverte), s’ils avaient l'intention d'arrêter l'utilisation de cigarettes électroniques à un moment donné, le classement des raisons motivant le début de l'utilisation de la cigarette électronique et la fréquence d'utilisation de la cigarette électronique dans certains lieux.

Pour mesurer les perceptions des avantages, des dangers et des risques de la cigarette électronique, on a demandé aux répondants de dire s'ils étaient d'accord, sur une échelle de 1 à 5, avec divers énoncés, 1 indiquant qu'ils n'étaient pas du tout d'accord et 5, qu'ils étaient tout à fait d'accord. Cette section contenait huit énoncés de type « les cigarettes électroniques améliorent ma santé » (avantage), « la cigarette électronique est inoffensive » (danger) et « il est approprié de faire usage de cigarette électronique en présence de membres de la famille ou d'amis qui ne fument pas » (risque).

\section{Variables}

On a demandé aux répondants combien de cigarettes par jour ils consommaient lorsqu'ils étaient fumeurs. Les réponses ont été réparties dans trois catégories : 20 ou moins, 21 à 40 et 41 ou plus. Étant donné qu'un paquet de cigarettes contient 20 cigarettes, une réponse de 41 ou plus impliquait de fumer plus de 2 paquets par jour. Les paquets-années ont été calculés en prenant le nombre total de cigarettes fumées par année en période de tabagisme, en le divisant par 20 et en multipliant le résultat obtenu par le nombre d'années de tabagisme. Les répondants ayant affirmé fumer 20 cigarettes ou moins par jour lorsqu'ils fumaient ont été classés dans la catégorie des fumeurs " légers " et ceux fumant plus de 21 cigarettes par jour, dans la catégorie des « gros » fumeurs. Les caractéristiques liées au mode de vie et les variables relatives à l'utilisation de la cigarette électronique portent sur l'ensemble de l'échantillon et sont stratifiées en fonction de la catégorisation du répondant en «fumeur léger » ou « gros fumeur ». Pour obtenir un nombre de réponses suffisant dans chaque catégorie, les variables « perception de l'état de santé général », «fréquence de la consommation d'alcool » et « fréquence de l'activité physique » ont été réduites à trois valeurs chacune.

Afin de mesurer la fréquence d'utilisation de la cigarette électronique, on a demandé aux répondants : « En moyenne, combien de temps dure une bouteille de liquide à vapoter? " et " Quel est le format de la bouteille (en $\mathrm{ml}$ )?». On leur a assigné une valeur pour la consommation quotidienne moyenne de liquide à vapoter en divisant le format de la bouteille par le nombre de jours nécessaires pour la consommer.

On a demandé aux répondants s'ils avaient ressenti, au cours des 2 heures suivant l'utilisation de la cigarette électronique, l'un des 14 effets secondaires énumérés, effets sélectionnés à partir d'un sondage international auprès des utilisateurs de cigarettes électroniques ${ }^{16}$. Les répondants ont aussi été invités à dire s'ils utilisaient « régulièrement », « parfois », « rarement » ou « jamais » les cigarettes électroniques dans certains lieux, notamment les restaurants, et dans quelle mesure les sept raisons suggérées pour l'utilisation de la cigarette électronique s'appliquaient à eux, en choisissant une réponse parmi « très », « assez », « un peu », « pas du tout important » ou « sans objet ».

Afin de déterminer si les caractéristiques des participants variaient en fonction de leur perception des dangers de la cigarette électronique, les réponses à l'énoncé « la cigarette électronique est inoffensive » ont été évaluées. Les répondants qui étaient d'accord ou tout à fait d'accord avec l'énoncé selon laquelle la cigarette électronique était inoffensive ont été classés dans la catégorie « inoffensive », les autres dans la catégorie « dommageable ». Pour obtenir suffisamment de réponses dans chaque catégorie, les variables liées au niveau de scolarité, au revenu familial, à la perception de l'état de santé général, à la fréquence de la consommation d'alcool et de l'activité physique et à l'utilisation de la cigarette électronique au travail ou à l'école ont été réduites à trois choix de réponses ou moins avant l'inclusion dans la régression logistique.

\section{Analyses statistiques}

Afin de caractériser les utilisateurs de cigarettes électroniques, nous avons calculé diverses fréquences, proportions, médianes et moyennes ainsi que des intervalles de confiance (IC) à $95 \%$, à l'aide de la version 22 du logiciel SPSS (IBM, Chicago, Illinois, États-Unis) ${ }^{24}$. Nous avons effectué des tests du chi carré de Pearson (non présentés) et calculé leurs valeurs $p$ à $95 \%$ pour déterminer tout écart significatif sur le plan statistique entre fumeurs légers et gros fumeurs (en période de tabagisme) en fonction des caractéristiques de leur mode de vie, de leurs raisons d'adopter la cigarette électronique, de leur façon d'en faire usage et des lieux où ils l'utilisaient. Nous avons construit des modèles de régression logistique pour évaluer la relation entre ces caractéristiques et la perception de l'innocuité de la cigarette électronique et nous avons calculé des rapports de cotes (RC) et leur IC à $95 \%$ ainsi que des RC non ajustés et ajustés selon le sexe et selon l'âge.

\section{Résultats}

Sur les 383 personnes qui ont répondu à l'invitation au sondage, 141 ont répondu « non » à la question leur demandant si elles avaient effectué un achat dans un commerce de cigarettes électroniques local (région d'Ottawa), ce qui fait qu'elles ont été exclues de l'étude.

Des 242 répondants restant, presque deux fois plus d'hommes $(\mathrm{n}=159)$ que de femmes ( $\mathrm{n}=83$ ) ont répondu au sondage en ligne. Lâge moyen des 
répondants était de 38,1 ans (plage : 16 à 70 ans). Plus de la moitié des répondants étaient mariés ou en union de fait (56,9\%), la majorité des répondants n'appartenaient pas à une minorité visible $(89,0 \%)$ et les deux tiers avaient terminé des études postsecondaires $(66,5 \%)$ (tableau 1$)$.

Près de la totalité des répondants avaient fumé 100 cigarettes ou plus au cours de leur vie $(97,9 \%)$ (tableau 1$)$. Le nombre moyen d'années de tabagisme était de 20,2 ans (plage : 2 à 50 ans), le nombre moyen de cigarettes fumées par jour en période de tabagisme était de 21 (plage : 1 à 75 cigarettes) et le nombre moyen de paquets-années était de 23,9 (plage : 0,20 à 122,85 paquets-années).

Davantage de fumeurs légers que de gros fumeurs ont ressenti des effets secondaires au cours des 2 heures suivant l'utilisation d'une cigarette électronique $(48,0 \%$ contre $35,0 \%, p=0,05$ ) (tableau 2). En revanche, davantage de gros fumeurs consommaient de l'alcool rarement (quelques fois par année) ou jamais $(44,7 \%$ contre $28,1 \%$ pour les fumeurs légers, $p=0,02$ ). Davantage de fumeurs légers ont appris l'existence des cigarettes électroniques d'abord par Internet $(18,8 \%$ contre $7,8 \%, p=0,02)$ alors que les gros fumeurs en ont d'abord entendu parler par la publicité ou un autre média $(15,5 \%$ contre $5,5 \%, p=0,02)$.

Les principales raisons ayant incité les répondants à faire usage de cigarettes électroniques étaient la volonté de cesser de fumer ou de réduire sa consommation de tabac. Le plus souvent, les répondants utilisaient la cigarette électronique chez eux, et $63,8 \%$ ont indiqué qu'ils vapotaient, régulièrement ou à l'occasion, au travail ou à l'école (tableau 2).

La presque totalité des répondants (96,6\%) faisaient usage de nicotine dans leurs cigarettes électroniques (tableau 2). Plus de la moitié des répondants n'ont signalé aucun des effets secondaires énumérés $(58,5 \%)$. Parmi les répondants ayant signalé des effets secondaires, 71 ont fait état de sécheresse ou d'irritation de la bouche ou de la gorge, 33 de toux, 23 de mal de tête et 17 d'étourdissements (données non présentées). Les effets secondaires moins fréquemment signalés étaient de la
TABLEAU 1

Caractéristiques sociodémographiques et antécédents de tabagisme des répondants au sondage $(N=242)$, Ottawa (Canada)

$\begin{array}{ccc}\text { Caractéristiques sociodémographiques } & \text { Nombre } & \text { Pourcentage } \\ \text { (n) } & (\%)\end{array}$

\section{Sexe}

Hommes

Femmes

Âge (ans)

Moins de 25

16,5

25 à 34

29,3

35 à 44

20,7

45 à 54

19,4

55 et plus

14,1

Situation de famille ${ }^{\mathrm{a}}$

Célibataire ou jamais marié

30,2

Séparé, divorcé ou veuf

12,9

Marié ou en union de fait

56,9

Minorité visible ${ }^{\mathrm{b}}$

Oui

11,0

Non

89,0

Employéc

Oui

83,7

Non

Niveau d'études ${ }^{c}$

Inférieur à l'école secondaire

Diplôme d'études secondaires

1,3

Diplôme d'études collégiales ou premier cycle universitaire

32,2

Deuxième cycle universitaire ou plus

60,5

Revenu familial $(\$)^{\mathrm{d}}$

Moins de 20000

20000 à 39999

11,7

40000 à 59999

14,8

60000 à 79999

18,9

80000 à 99999

20,4

100000 ou plus

17,3

déjà fumé 100 cigarettes et plus au cours de sa vie ${ }^{\mathrm{e}}$

16,8

Oui

Non

97,9

Consommation quotidienne de cigarettes (dans les périodes de tabagisme) $^{\mathrm{f}}$
20 (1 paquet) ou moins
21 à 40 (2 paquets)
41 (2 paquets) ou plus
Jamais fumé

54,2

39,0

4,7

2,1 
TABLEAU 2

Caractéristiques liées au mode de vie et utilisation de la cigarette électronique chez les « fumeurs légers » et chez les « gros fumeurs » (en période de tabagisme; $\mathrm{N}=242 \%$, 2015, Ottawa (Canada)

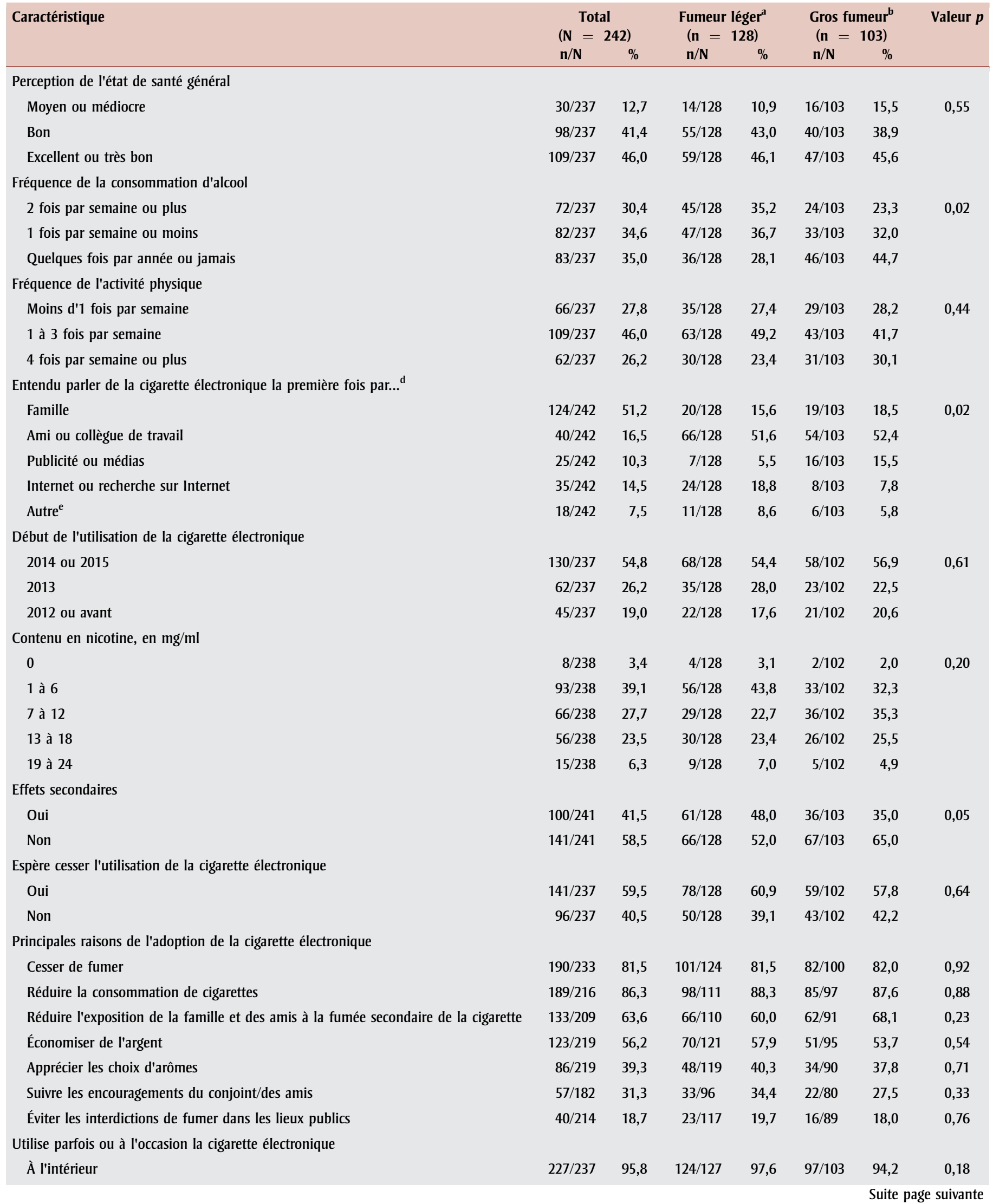


TABLEAU 2 (suite)

Caractéristiques liées au mode de vie et utilisation de la cigarette électronique chez les «fumeurs légers » et chez les " gros fumeurs » (en période de tabagisme; $\mathbf{N}=242^{\circ}$ ), 2015, Ottawa (Canada)

\begin{tabular}{|c|c|c|c|c|c|c|c|}
\hline \multirow[t]{2}{*}{ Caractéristique } & \multirow{2}{*}{\multicolumn{2}{|c|}{$\begin{array}{c}\text { Total } \\
(\mathrm{N}=242)\end{array}$}} & \multirow{2}{*}{\multicolumn{2}{|c|}{$\begin{array}{l}\text { Fumeur léger }^{a} \\
(\mathbf{n}=128)\end{array}$}} & \multirow{2}{*}{\multicolumn{2}{|c|}{$\begin{array}{l}\text { Gros fumeur } \\
(n=103)\end{array}$}} & \multirow[t]{2}{*}{ Valeur $p$} \\
\hline & & & & & & & \\
\hline À l'extérieur & $221 / 235$ & 94,0 & $118 / 127$ & 92,9 & 97/102 & 95,1 & 0,49 \\
\hline À l'intérieur, chez des membres de la famille & $150 / 234$ & 64,1 & $77 / 126$ & 61,1 & 70/101 & 69,3 & 0,20 \\
\hline Au travail ou à l'école & $146 / 229$ & 63,8 & 79/124 & 63,7 & $65 / 99$ & 65,7 & 0,76 \\
\hline Dans les restaurants & $42 / 235$ & 17,9 & $20 / 126$ & 15,9 & $20 / 102$ & 19,6 & 0,46 \\
\hline Dans les transports en commun & $34 / 233$ & 14,6 & $21 / 126$ & 16,7 & $12 / 100$ & 12,0 & 0,32 \\
\hline
\end{tabular}

${ }^{a}$ Les répondants ayant déclaré avoir fumé 20 cigarettes ou moins par jour (en période de tabagisme) ont été considérés comme des « fumeurs légers ».

b Les répondants ayant déclaré avoir fumé 21 cigarettes ou plus par jour (en période de tabagisme) ont été considérés comme des « gros fumeurs ».

c II y a eu 11 réponses manquantes ou de non-fumeurs sur la question du nombre de cigarettes fumées par jour (en période de tabagisme).

${ }^{\mathrm{d}}$ Le dénominateur $(\mathrm{N})$ pour chacune des variables exclut les réponses manquantes ou sans objet.

e Dans la catégorie « Autre », la réponse « un médecin » a été fournie par 3 fumeurs légers et 3 gros fumeurs (en période de tabagisme).

somnolence $(\mathrm{n}=10)$, des ulcères buccaux ou de la langue $(n=7)$, de la fatigue $(\mathrm{n}=5)$, des palpitations cardiaques $(\mathrm{n}=4)$, des allergies $(\mathrm{n}=4)$, des douleurs à la poitrine $(\mathrm{n}=3)$, des difficultés respiratoires $(n=3)$, des saignements de nez $(n=2)$, des saignements des gencives $(n=2)$ et du stress ( $\mathrm{n}=1$ ) (données non présentées).

Plus de la moitié des personnes interrogées $(59,5 \%)$ ont mentionné qu'elles espéraient arrêter l'utilisation de la cigarette électronique un jour (tableau 2).

Le temps moyen consacré à l'usage de la cigarette électronique était d'environ 5 minutes par séance et l'utilisation moyenne de liquide à vapoter par jour était de 2,15 ml (données non présentées). Cela signifie qu'il fallait en moyenne 14 jours aux participants pour terminer une bouteille de liquide à vapoter de $30 \mathrm{ml}$. Bien que les types de dispositifs de cigarettes électroniques utilisés aient considérablement varié, les dispositifs de taille moyenne, les dispositifs à réservoir ( « tank ») et les dispositifs Joyetech eGo-C avec cartouches de liquide à vapoter étaient les plus utilisés. Trente-quatre répondants ont indiqué qu'ils utilisaient une combinaison de composantes de cigarettes électroniques de différentes marques.

Les trois quarts des répondants étaient tout à fait d'accord avec l'énoncé selon lequel la cigarette électronique constitue une façon efficace d'arrêter de fumer (71,7\%) et celui selon lequel la cigarette électronique les aidait à améliorer leur santé (75,5 \%), $60,1 \%$ étaient d'accord ou tout à fait d'accord avec l'énoncé selon lequel la cigarette électronique est inoffensive (tableau 3; certaines données non présentées). Les répondants ayant signalé n'avoir ressenti aucun des effets secondaires énumérés étaient environ 3 fois plus nombreux que ceux ayant signalé un effet secondaire ou plus à ne percevoir aucun danger dans la cigarette électronique $(\mathrm{RC}=3,17$; IC à $95 \%: 1,75$ à 5,73) (tableau 4).

\section{Analyse}

Divers organismes de santé publique, instances de réglementation et chercheurs ont suggéré que l'adoption de la cigarette électronique par les personnes n'ayant jamais fumé comme par les fumeurs pourraient nuire à la lutte contre le tabagisme $^{1,11}$. Ils considèrent que les personnes n'ayant jamais fumé qui utilisent la cigarette électronique pourraient être exposées à des risques pour la santé auxquels elles n'auraient pas été exposées par ailleurs et que les fumeurs faisant usage de la cigarette électronique pourraient ne pas réduire leurs risques autant qu'ils le croient.

À l'instar des autres études disponibles, la majorité des répondants avaient déjà fumé des cigarettes ${ }^{12-16}$. Une étude récente portant sur 19414 répondants recrutés par l'entremise d'un site Internet pour une recherche sur la cigarette électronique a révélé que 99,5 \% des utilisateurs fumaient ou avaient déjà fumé ${ }^{16}$. Selon notre étude, qui utilise comme critère de tabagisme d'avoir fumé 100 cigarettes ou plus au cours de sa vie, ce sont 97,9 \% des répondants qui fumaient ou avaient déjà fumé. Notre sondage n'a cependant pas déterminé si l'usage du tabac avait précédé l'utilisation de la cigarette électronique ou s'il se poursuivait de façon concomitante.

Les cigarettes électroniques pouvant être constituées de plusieurs composantes, les marques, les types et les façons de les modifier varient considérablement ${ }^{4}$. Cette variabilité des marques et des modifications a rendu difficile le classement des types de cigarettes électroniques les plus utilisés. Néanmoins, le dispositif que nos répondants ont mentionné utiliser le plus fréquemment est le Joyetech eGo-C, le même que celui relevé dans l'étude de Dawkins et collab $^{13}$. Outre l'existence de différents types de dispositifs et de modifications possibles, il faut noter que la fabrication des cigarettes électroniques est peu réglementée, ce qui est susceptible d'entraîner des problèmes d'assurance de la qualité ${ }^{2}$. Les écarts observés dans notre petit échantillon local sont le signe d'une variabilité potentielle plus grande parmi les dispositifs de cigarettes électroniques en général. En raison de ces écarts, il peut 
TABLEAU 3

Perceptions des participants au sondage relativement aux avantages, aux dangers et aux risques de la cigarette électronique $\left(n=233^{a}\right), 2015$, Ottawa $(\text { Canada })^{28}$

\begin{tabular}{|c|c|c|}
\hline Énoncé de perception & $\begin{array}{c}\text { Moyenne } \\
\text { (IC à } 95 \%)^{\text {b }}\end{array}$ & $\begin{array}{c}\text { Tout à fait } \\
\text { d'accord (n, \%) }\end{array}$ \\
\hline La cigarette électronique m'a aidé à améliorer ma santé & $4,58(4,46$ à 4,69$)$ & $176(75,5)$ \\
\hline La cigarette électronique est une bonne façon d'arrêter de fumer & $4,55(4,43$ à 4,66$)$ & $167(71,7)$ \\
\hline Ma famille ou mes amis me soutiennent dans mon utilisation de la cigarette électronique & $4,33(4,20$ à 4,46) & $140(60,1)$ \\
\hline $\begin{array}{l}\text { Il est approprié de faire usage de la cigarette électronique en présence de membres de la famille ou d'amis qui } \\
\text { ne fument pas }{ }^{c}\end{array}$ & $3,74(3,59$ à 3,88) & $70(30,2)$ \\
\hline Il est approprié de faire usage de cigarette électronique en présence d'enfants & $2,86(2,68$ à 3,03) & $38(16,3)$ \\
\hline La cigarette électronique devrait être soumise aux mêmes restrictions que la cigarette au tabac & $1,65(1,50$ à 1,81$)$ & $17(7,3)$ \\
\hline La cigarette électronique est aussi dommageable que la cigarette au tabac & $1,19(1,10$ à 1,28$)$ & $4(1,7)$ \\
\hline
\end{tabular}

Abréviation : IC, intervalle de confiance.

a 233 participants ont répondu aux questions sur les perceptions à la fin du sondage.

${ }^{\text {b }}$ Perception moyenne sur une échelle de 1 à 5,1 indiquant que le répondant n'était pas du tout d'accord et 5, qu'il était tout à fait d'accord.

c 1 réponse manquante pour cette variable.

être difficile de tirer des conclusions à propos de l'innocuité des dispositifs de cigarettes électroniques ${ }^{25}$.

Les produits contenant de la nicotine sont réglementés en vertu de la Loi sur les aliments et drogues et une autorisation de Santé Canada est nécessaire pour les vendre et pour en faire la publicitée ${ }^{26}$. Bien que les dispositifs de cigarettes électroniques avec produits contenant de la nicotine ne soient pas encore autorisés au Canada, nous avons constaté que $96,6 \%$ des répondants de notre étude utilisaient un liquide à vapoter contenant de la nicotine. Si nos résultats proviennent seulement d'un échantillon de commodité, des études à plus grande échelle ont révélé que ce sont $96 \%$ et plus de leurs répondants qui utilisaient un liquide à vapoter contenant de la nicotine ${ }^{13,16}$. Nos résultats pourraient avoir été influencés par le fait que nous avons recruté nos répondants par l'entremise de commerces de cigarettes électroniques locaux qui, à notre connaissance, ne vendaient pas de cigarettes électroniques jetables.

De plus, notre sondage n'a recueilli de renseignements que sur l'usage actuel de nicotine. Il est possible que les personnes qui vapotent pour réduire ou cesser leur consommation de tabac réduisent ou cessent aussi l'utilisation de la nicotine dans leurs cigarettes électroniques avec le temps.

Nous avons noté que le contenu en nicotine indiqué sur l'étiquette ne correspondait pas toujours au contenu en nicotine réel des liquides à vapoter ${ }^{2,4}$. Des vérifications auprès des détaillants de cigarettes électroniques pourraient fournir des renseignements importants sur l'accessibilité réelle à des liquides à vapoter contenant de la nicotine.

Par rapport à une étude réalisée aux ÉtatsUnis selon laquelle $71 \%$ des utilisateurs quotidiens de cigarettes électroniques vapotaient au travail, $43 \%$ dans les bars ou cafés et $15 \%$ dans les transports en commun ${ }^{14}$, environ $64 \%$ des répondants de notre sondage ont déclaré faire usage de cigarette électronique régulièrement ou à l'occasion au travail ou à l'école, et $15 \%$ dans les transports en commun. La Loi de 2015 pour des choix plus sains, qui a reçu la Sanction royale le 28 mai 2015, va réglementer de nombreux aspects de l'utilisation de la cigarette électronique en Ontario, notamment les lieux où elle pourra être utilisée $e^{27}$. Notre sondage a révélé qu'il est fait usage de cigarette électronique dans des lieux où la cigarette est bannie, exposant potentiellement les autres personnes aux vapeurs secondaires de ces cigarettes électroniques. Les dispositions de la Loi de 2015 sur les cigarettes électroniques concernant leur utilisation dans les lieux publics ne sont pas encore entrées en vigueur, mais leur mise en place et leur application pourraient modifier l'utilisation de la cigarette électronique dans les lieux publics. En l'absence de cette réglementation, les organismes peuvent autoréglementer l'utilisation de la cigarette électronique, quoiqu'on ne sache pas exactement dans quelle mesure cette autoréglementation est pratiquée, suivie et appliquée.

Les effets secondaires les plus fréquemment signalés par les répondants (bouche ou gorge sèche ou irritée, toux) sont souvent relevés dans la documentation médicale ${ }^{14,17,28,29}$. Ce n'est pas surprenant, car la glycérine et le propylèneglycol en aérosol, qui sont les principaux ingrédients du liquide à vapoter, sont associés à des irritations de la bouche et de la gorge. Il est possible que ces effets secondaires se résorbent avec le temps (la moitié des participants utilisaient les cigarettes électroniques depuis moins de 14 mois au moment du sondage $)^{16,29}$. Certains répondants ont signalé des effets sur la santé plus graves : 4 ont noté des palpitations cardiaques et 3 des douleurs à la poitrine. Dans une synthèse sur les effets indésirables liés potentiellement à la cigarette électronique, $\operatorname{Chen}^{28}$ a noté que des douleurs à la poitrine et une accélération de la fréquence cardiaque avaient 
TABLEAU 4

Rapports de cotes pour les caractéristiques des participants au sondage percevant la cigarette électronique comme inoffensive (n = 233), 2015, Ottawa (Canada)

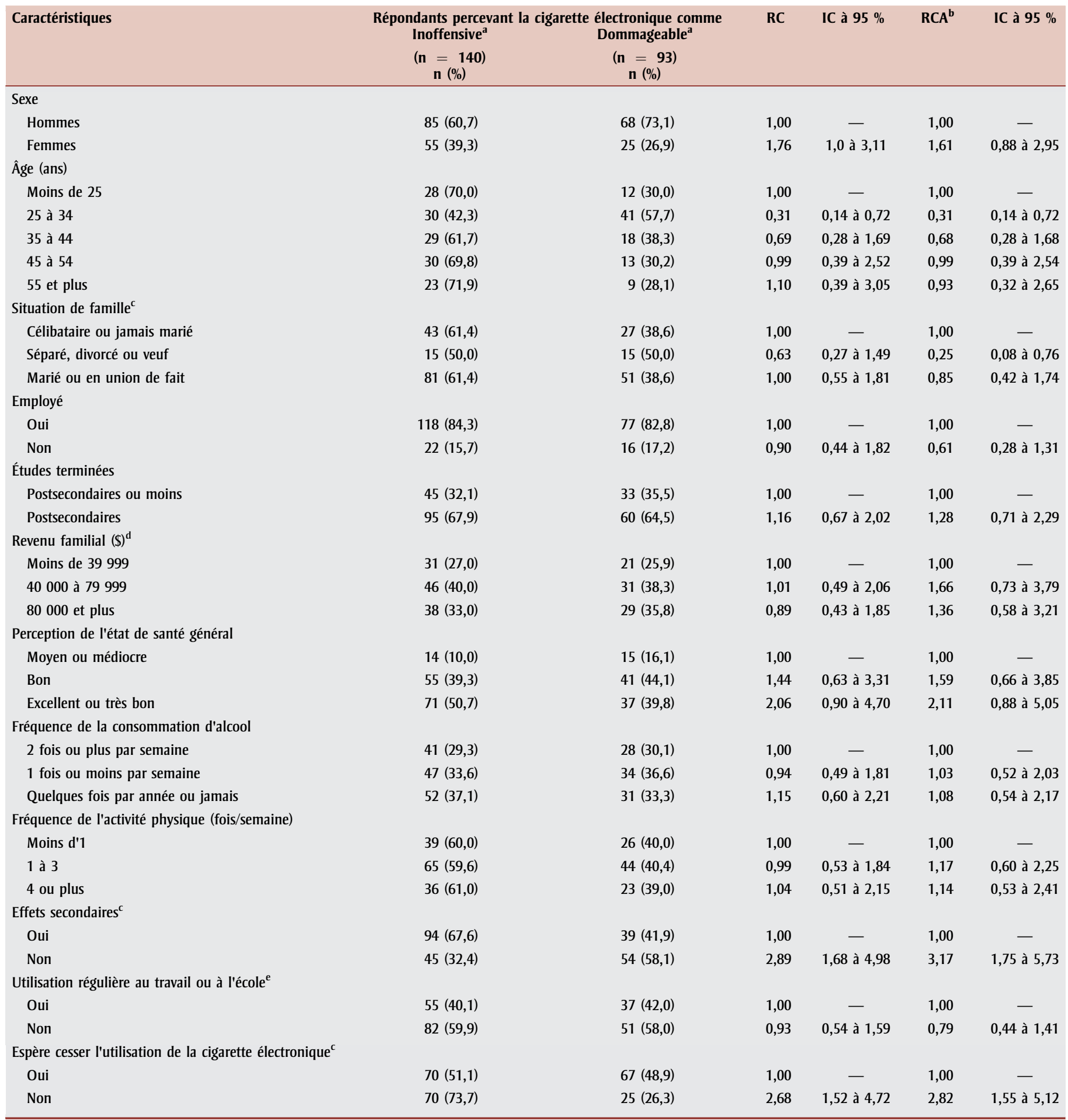

Abréviations : IC, intervalle de confiance; RC, rapport de cotes; RCA, rapport de cotes ajusté.

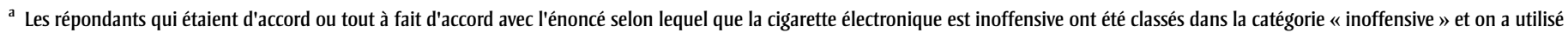
la catégorie « dommageable » pour les autres réponses.

b Rapports de cotes ajustés selon l'âge et le sexe.

c 1 réponse manquante.

d 37 réponses manquantes ou " préfère ne pas répondre ».

e 8 réponses manquantes. 
été signalées à la Food and Drug Administration.

Plusieurs études ont mentionné que les utilisateurs ne perçoivent en général pas la cigarette électronique comme étant totalement inoffensive, mais tous la considèrent comme moins nocive que les cigarettes ${ }^{14,16,17}$. Plus de la moitié de notre échantillon (60,1\%) percevait la cigarette électronique comme inoffensive, et cette tendance était plus marquée chez les femmes (données non présentées). Il n'est donc pas surprenant que les personnes n'ayant signalé aucun des 14 effets secondaires énumérés aient été plus nombreuses à percevoir la cigarette électronique comme inoffensive (tableau 4). La perception de la cigarette électronique comme inoffensive pourrait jouer un rôle dans la décision d'une personne d'en faire usage et dans la mesure dans laquelle elle en ferait usage en présence d'autres personnes.

\section{Points forts et limites}

Notre étude vient enrichir la littérature, rare, sur les caractéristiques des utilisateurs de cigarettes électroniques, en fournissant des renseignements détaillés sur l'utilisation de la cigarette électronique, les lieux où elle est utilisée et les perceptions qu'en ont ses utilisateurs. Ce sondage fournit des observations qui, couplées à celles d'autres études, sont susceptibles de guider les priorités et les orientations de recherche des décideurs. Il peut également se révéler utile pour les enquêtes à venir sur les utilisateurs de cigarettes électroniques.

Notre capacité de généralisation des caractéristiques et des perceptions des répondants à notre sondage est limitée en raison de la petite taille de l'échantillon $(\mathrm{n}=242)$. Il est possible que les caractéristiques des personnes ayant acheté des cigarettes électroniques dans un commerce d'Ottawa diffèrent de celles des personnes qui achètent des cigarettes électroniques ailleurs (dans des stationsservices, en ligne, etc.) ainsi que des utilisateurs de cigarettes électroniques d'autres régions. L'utilisation d'un échantillon de commodité limitant ainsi la généralisation potentielle des constatations aux utilisateurs de cigarettes électroniques canadiens, les constatations faites ici doivent être interprétées avec prudence.

Il pourrait y avoir un biais lié aux répondants, les personnes qui perçoivent la cigarette électronique de façon positive pouvant avoir été davantage motivées à répondre à un sondage portant sur la cigarette électronique que celles en ayant une perception moins favorable. De plus, le sondage ayant été effectué au cours des deux premiers mois de l'année, il pourrait offrir une représentation disproportionnée de personnes ayant pris en début d'année la bonne résolution d'arrêter de fumer. Enfin, le sondage ayant été administré en anglais, les personnes dont la langue dominante n'est pas l'anglais pourraient être moins bien représentées. Notons qu'il fallait disposer d'une connexion Internet pour participer (ce qui ne constitue probablement pas un obstacle en soi).

Même si le sondage recueillait des renseignements sur les antécédents de tabagisme des répondants, il ne comportait aucune question sur les habitudes actuelles de tabagisme, ce qui fait que nous n’avons donc pas évalué l'utilisation concomitante (simultanée) de la cigarette électronique et de la cigarette.

\section{Conclusion}

En dépit de ces limites, notre sondage a révélé plusieurs faits intéressants à propos des vapoteurs de la région d'Ottawa. Nous avons constaté que la majorité des répondants de cet échantillon de commodité d'utilisateurs de cigarettes électroniques avaient des antécédents de tabagisme, utilisaient du liquide à vapoter contenant de la nicotine et avaient une perception favorable de la cigarette électronique. La réduction ou l'arrêt de la consommation de tabac faisaient partie des raisons invoquées très importantes pour avoir adopté la cigarette électronique, et plus de la moitié des répondants ont mentionné avoir l'espoir de cesser d'utiliser la cigarette électronique un jour. D’autres sondages vont être nécessaires pour caractériser le profil des utilisateurs de cigarettes électroniques dans d'autres régions canadiennes, et en fonction d'autres facteurs sociodémographiques et culturels. Nous espérons que nos constatations pourront être utiles dans les enquêtes à venir sur l'utilisation de la cigarette électronique, et qu'elles fourniront une aide efficace aux décideurs lors de l'établissement de leurs priorités en recherche.

\section{Remerciements}

Nous sommes reconnaissants à la Faculté des sciences de la santé de l'Université Carleton pour le financement de cette étude. Nous tenons aussi à remercier l'Unité de recherche sur le tabac de Santé Canada pour la relecture du sondage et Scott Weichenthal pour ses conseils sur la méthodologie de notre étude.

\section{Références}

1. Grana R, Benowitz N, Glantz SA. Background paper on e-cigarettes (electronic nicotine delivery systems) [Internet]. San Francisco (CA) University of California 2013 http://arizonansconcernedaboutsmoking.com/ 2013 12e-cig_report.pdfPDF téléchargeable à partir du lien : http://arizonansconcernedaboutsmoking.com/2013 12e-cig_report.pdf.

2. Grana R, Benowitz N, Glantz SA. E-cigarettes: a scientific review Circulation. 2014;129(19):1972-1986

3. Callahan-Lyon P. Electronic cigarettes: human health effects. Tob Control. 2014;23 Suppl 2: ii36-40. doi : 10.1136/tobaccocontrol-2013 -051470 .

4. Harrell PT, Simmons VN, Correa JB, Padhya TA, Brandon TH. Electronic nicotine delivery systems ("e-cigarettes"): review of safety and smoking cessation efficacy Otolaryngol Head Neck Surg. 2014;151(3) 381-393. doi: 10.1177/0194599814536847.

5. Czogala J, Goniewicz ML, Fidelus B, Zielinska-Danch W, Travers MJ, Sobczak A. Secondhand exposure to vapors from electronic cigarettes. Nicotine Tob Res. 2014;16(6):655-662. doi: 10.1093/ntr/ntt203.

6. McAuley TR, Hopke PK, Zhao J, Babaian S. Comparison of the effects of e-cigarette vapor and cigarette smoke on indoor air quality. Inhal Toxicol. 2012;24(12):850-857. doi: 10.3109/08958378.2012.724728. 
7. Pisinger C, Dossing M. A systematic review of health effects of electronic cigarettes. Prev Med. 2014;69:248-260. doi: 10.1016 /j.ypmed.2014.10.009.

8. Breland AB, Spindle T, Weaver M, Eissenberg T. Science and electronic cigarettes: current data, future needs. J Addict Med. 2014;8(4):223-233. doi: 10.1097/ADM.0000000000000049.

9. Ayers JW, Ribisl KM, Brownstein JS. Tracking the rise in popularity of electronic nicotine delivery systems (electronic cigarettes) using search query surveillance. Am J Prev Med. 2011;40(4):448-453. doi: 10.1016 /j.amepre.2010.12.007.

10. Cobb NK, Byron MJ, Abrams DB, Shields PG. Novel nicotine delivery systems and public health: the rise of the "e-cigarette". Am J Public Health. 2010;100(12):2340-2342. doi: 10.2105/AJPH.2010.199281.

11. Glynn TJ. E-cigarettes and the future of tobacco control. CA Cancer J Clin. 2014; 64(3):164-168. doi: 10.3322/caac.21226.

12. Czoli CD, Hammond D, White CM. Electronic cigarettes in Canada: prevalence of use and perceptions among youth and young adults Can J Public Health. 2014;105(2):e97-102.

13. Dawkins L, Turner J, Roberts A, Soar K. 'Vaping' profiles and preferences: an online survey of electronic cigarette users. Addiction. 2013;108(6):1115-1125. doi: 10.1111/add.12150.

14. Etter JF, Bullen C. Electronic cigarette: users profile, utilization, satisfaction and perceived efficacy Addiction. 2011;106(11) 2017-2028. doi: 10.1111/j.0443-0443.2011. 03505.x

15. King BA, Alam S, Promoff G, Arrazola R, Dube SR. Awareness and ever-use of electronic cigarettes among U.S. adults, 20102011. Nicotine Tob Res. 2013;15(9):16231627. doi: $10.1093 / \mathrm{ntr} / \mathrm{ntt} 013$.

16. Farsalinos KE, Romagna G, Tsiapras D, Kyrzopoulos S, Voudris V. Characteristics, perceived side effects and benefits of electronic cigarette use: a worldwide survey of more than 19,000 consumers Int J Environ Res Public Health. 2014;11(4):4356-4373. doi: 10.3390/ijerph110404356.
17. Goniewicz ML, Lingas EO, Hajek P. Patterns of electronic cigarette use and user beliefs about their safety and benefits: an internet survey. Drug Alcohol Rev. 2013;32(2):133-140. doi: 10.1111/j.1465-3362.2012.00512.x.

18. McRobbie H, Bullen C, Hartmann-Boyce J, Hajek P. Electronic cigarettes for smoking cessation and reduction. Cochrane Database Syst Rev. 2014;12:CD010216. doi: 10.1002/14651858.CD010216.pub2.

19. Rahman MA, Hann N, Wilson A, Mnatzaganian G, Worrall-Carter L. E-cigarettes and smoking cessation: evidence from a systematic review and meta-analysis. PLoS One. 2015;10(3):e0122544 doi: 10.1371/journal.pone.0122544.

20. Adkison SE, O'Connor RJ, Bansal-Travers M, Hyland A, Borland R, Yong HH et collab. Electronic nicotine delivery systems: international tobacco control four-country survey. Am J Prev Med. 2013;44(3):207-215. doi: 10.1016/j.amepre.2012.10.018.

21. Reid JL, Rynard VL, Czoli CD, Hammond D. Who is using e-cigarettes in Canada? Nationally representative data on the prevalence of e-cigarette use among Canadians. Prev Med. 2015;81:180-183. doi: 10.1016/j.ypmed.2015.08.019.

22. Qualtrics Research Suite [Internet]. Provo (UT): Qualtrics L; 2015 [consulté le $1^{\mathrm{er}}$ décembre 2014]. Consultable en ligne à partir de la page : http://www.qualtrics .com/research-suite/

23. Statistique Canada. Enquête sur la santé dans les collectivités canadiennes (ESCC) Composante annuelle : Complément au Guide de l'utilisateur - Collection de fichiers de microdonnées à grande diffusion 2012 et 2011-2013. Ottawa (Ont.) : Gouvernement du Canada; 2013.

24. SPSS Statistics Standard [Internet]. Chicago (IL): IBM; 2014. Consultable en ligne à partir de la page : http://www-03.ibm.com /software/products/en/spss-stats-standard

25. Harrell PT, Marquinez NS, Correa JB et collab. Expectancies for cigarettes, e-cigarettes, and nicotine replacement therapies among e-cigarette users (aka vapers). Nicotine Tob Res. 2015;17(2):193-200. doi: 10.1093/ntr /ntul49.
26. Santé Canada. Médicaments et produits de santé. Avis - À toutes les personnes qui souhaitent importer, annoncer ou vendre des cigarettes électroniques au Canada [communiqué de presse] [Internet]. Ottawa (Ont.) : Santé Canada; 2009 [consulté le $1^{\text {er }}$ juin 2015]. Consultable en ligne à la page http://www.hc-sc.gc.ca/dhp-mps/prodpharma /applic-demande/pol/notice_avis_e-cig-fra.php

27. Loi de 2015 sur les cigarettes électroniques; Projet de loi 45, Loi de 2015 pour des choix plus sains : Loi visant à améliorer la santé publique par l'édiction de la Loi de 2015 pour des choix santé dans les menus et de la Loi de 2015 sur les cigarettes électroniques et la modification de la Loi favorisant un Ontario sans fumée [Internet]. Toronto (Ont.) : Assemblée législative de l'Ontario; 2015 [consulté le $1^{\mathrm{er}}$ juin 2015]. Consultable en ligne à la page : http://www.ontla.on.ca /web/bills/bills_detail.do?locale $=$ fr\&BillID $=3080$

28. Chen IL. FDA summary of adverse events on electronic cigarettes. Nicotine Tob Res. 2013;15(2):615-616. doi: 10.1093/ntr/nts145.

29. Polosa R, Caponnetto P, Morjaria JB, Papale G, Campagna D, Russo C. Effect of an electronic nicotine delivery device (e-Cigarette) on smoking reduction and cessation: a prospective 6-month pilot study. BMC Public Health. 2011;11:786 doi: 10.1186/1471-245811-786. 